$67^{\text {th }}$ International Astronautical Congress (IAC), Adelaide, Australia, 25-29 September 2017.

Copyright $@ 2017$ by the authors. Published by the International Astronautical Federation (IAF), with permission and released to the IAF to publish in all forms.

IAC-17-C4,6,5,x41810

\title{
SYSTEM ANALYSIS AND TEST-BED FOR AN ATMOSPHERE-BREATHING ELECTRIC PROPULSION SYSTEM USING AN INDUCTIVE PLASMA THRUSTER
}

\author{
Francesco Romano $^{\mathrm{d}^{*}}$, Tilman Binder ${ }^{\mathrm{d}}$, Georg H. Herdrich ${ }^{\mathrm{d}}$, Peter C.E. Roberts ${ }^{\mathrm{a}}$, Silvia Rodriguez- \\ Donaire ${ }^{\mathrm{e}}$, Daniel Garcia-Almiñana ${ }^{\mathrm{e}}$, Nicholas H. Crispa ${ }^{\mathrm{a}}$, Steve Edmondson ${ }^{\mathrm{a}}$, Sarah J. Haigh ${ }^{\mathrm{a}}$, Rachel E. \\ Lyons $^{\mathrm{a}}$, Vitor T.A. Oiko ${ }^{\mathrm{a}}$, Katharine L. Smith ${ }^{\mathrm{a}}$, Jonathan Becedas ${ }^{\mathrm{b}}$, Gerardo González ${ }^{\mathrm{b}}$, Irene Vázquez ${ }^{\mathrm{b}}$, \\ Álvaro Braña ${ }^{b}$, Kelly Antonini ${ }^{c}$, Kristian Bayc, Leonardo Ghizonic ${ }^{c}$, Victor Jungnell ${ }^{c}$, Jonas Morsbølc, Adam \\ Boxberger $^{\mathrm{d}}$, Stefanos Fasoulas ${ }^{\mathrm{d}}$, Dhiren Kataria ${ }^{\mathrm{f}}$, Mark Davidson ${ }^{\mathrm{g}}$, Ron Outlaw ${ }^{\mathrm{g}}$, Badia Belkouchi ${ }^{\mathrm{h}}$, Alexis \\ Conte $^{\text {h }}$, Jose Santiago Perez ${ }^{\text {h }}$, Rachel Villain ${ }^{\text {h }}$, Barbara Heißerer ${ }^{\mathrm{i}}$, Ameli Schwalber ${ }^{\mathrm{i}}$ \\ a The University of Manchester, Oxford Road, Manchester, M13 9PL, United Kingdom \\ ${ }^{\mathrm{b}}$ Elecnor Deimos Satellite Systems, Calle Francia 9, 13500 Puertollano, Spain \\ c GomSpace AS, Langagervej 6, 9220 Aalborg East, Denmark \\ d Institute of Space Systems (IRS), University of Stuttgart, Pfaffenwaldring 29, 70569 Stuttgart, Germany, \\ romano@irs.uni-stuttgart.de \\ ${ }^{\mathrm{e}}$ UPC-BarcelonaTECH, Carrer de Colom 11, 08222 Terrassa, Barcelona, Spain \\ ${ }^{\mathrm{f}}$ Mullard Space Science Laboratory (UCL), Holmbury St. Mary, Dorking, RH5 6NT, United Kingdom \\ g The Tech ToyBox, 2153 SE Hawthorne Road, Gainesville, FL 32641, USA \\ ${ }^{\mathrm{h}}$ Euroconsult, 86 Boulevard de Sébastopol, 75003 Paris, France \\ ${ }^{i}$ Concentris Research Management GmbH, Ludwigstraße 4, D-82256 Fürstenfeldbruck, Germany
}

\begin{abstract}
Challenging space mission scenarios include those in very low Earth orbits, where the atmosphere creates significant drag to the S/C and forces their orbit to an early decay. For drag compensation, propulsion systems are needed, requiring propellant to be carried on-board. An atmosphere-breathing electric propulsion system (ABEP) ingests the residual atmosphere through an intake and uses it as propellant for an electric thruster. Theoretically applicable to any planet with atmosphere, the system might allow drag compensation for an unlimited time without carrying propellant. A new range of altitudes for continuous operation would become accessible, enabling new scientific missions while reducing costs. Preliminary studies have shown that the collectible propellant flow for an ion thruster (in LEO) might not be enough, and that electrode erosion due to aggressive gases, such as atomic oxygen, will limit the thruster's lifetime. In this paper we introduce the use of an inductive plasma thruster (IPT) as thruster for the ABEP system as well as the assessment of this technology against its major competitors in VLEO (electrical and chemical propulsion). IPT is based on a small scale inductively heated plasma generator IPG6-S. These devices have the advantage of being electrodeless, and have already shown high electric-to-thermal coupling efficiencies using $\mathrm{O}_{2}$ and $\mathrm{CO}_{2}$ as propellant. A water cooled nozzle has been developed and applied to IPG6-S. The system analysis is integrated with IPG6-S equipped with the nozzle for testing to assess mean mass-specific energies of the plasma plume and estimate exhaust velocities.
\end{abstract}

Keywords: ABEP, IPG, IPT, VLEO, RAM-EP

Acronyms/Abbreviations

ABEP: Atmosphere-Breathing Electric Propulsion

IPT: Inductive Plasma Thruster

IPG: Inductively heated Plasma Generator

VLEO: Very Low Earth Orbit

\section{Introduction}

Missions in LEO may open new market opportunities for weather forecasting, monitoring of oceanic currents, polar ice caps, fires, agriculture, and military and civil surveillance services. Recently ESA mission GOCE has ended, providing detailed information of Earth's geomagnetic field by orbiting as low as $229 \mathrm{~km}$ [1] using RIT as EP. Missions at low altitudes are limited in mission lifetime due to aerodynamic drag, caused by momentum exchange between the residual atmosphere particles and the $S / C$, requiring an efficient propulsion system that compensates the drag. Such low altitudes would allow simpler and smaller platforms, meaning lower costs, as well as ensuring self de-orbiting at the end of the mission [2]. For such missions, the maximum mission lifetime of a $\mathrm{S} / \mathrm{C}$ is a mission design driver that depends on the amount of drag that the propulsion system can compensate, and, second, for how long. These two are dependent on the propulsion system efficiency, on the amount of propellant carried on board, and on the generated of drag. Besides, businesses interest lies in the value they can get from the investment done. For that 
reason, the new value proposition that ABEP propulsion systems delivers to satellite launching businesses is extremely important, since advantages of EP systems like high specific impulse, capability to extend mission lifetime, in this specific case therefore enabling drag compensation in VLEO (ABEP), and cost-benefit analysis are key for launching such missions.

\section{Atmosphere-Breathing Electric Propulsion}

Atmosphere-Breathing Electric Propulsion System (ABEP) is a promising strategy to efficiently compensate the drag in orbit, enabling longer mission lifetime in VLEO and reducing propellant mass requirement. An ABEP system provides thrust to the spacecraft by collecting the residual atmosphere of the planet at low altitude orbits using a specially designed intake, and utilizing it as propellant for an electric thruster. The concept is to extend the spacecraft's lifetime by eliminating the need to carry propellant on-board. Providing a virtually unlimited amount of propellant, the lifetime- limiting factor is no longer the amount of propellant carried on-board, but the durability of the other satellite's subsystems. Eliminating the need of carry propellant into orbit brings the benefit of reduced launch mass as long as the mass of the ABEP system is less than that of a conventional propulsion system (including the propellant). However, the intake mass is yet to be determined, but might increase as the design altitude reduces. As a result, it is possible to use less powerful launch vehicles, reducing costs. Alternatively, the payload mass can be increased. In this paper, a system analysis for ABEP is proposed, where an inductive plasma thruster (IPT) is used as EP. IPT are electrodeless devices based on inductively heated plasma generators (IPG), therefore eliminating the performance degradation issue typical of RIT and HET, allowing a wide range of propellant to be used and, moreover, removing the need of a neutralizer. The concepts is shown within Fig. 1.

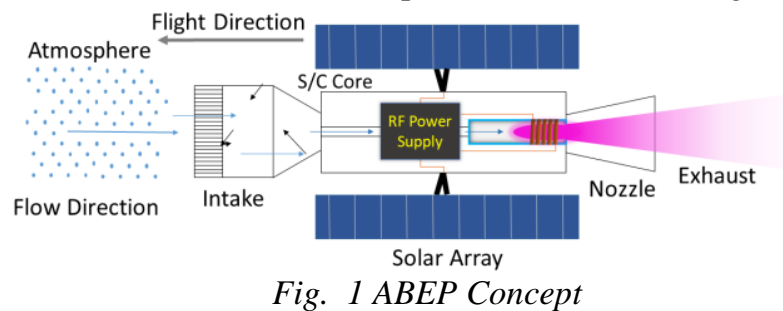

IRS has gathered several decades of experience in the development, operation, characterization and qualification of various plasma sources. Among them are steady state self-field and applied field magnetoplasmadynamic (MPD) sources, thermal arcjet devices, IPG and hybrid plasma systems [3], [4], [5]. These plasma systems are in application for aerothermodynamics testing, heat shield material characterization [6], [7], [8], [9], [10], [11], electric space propulsion [12], [13], [14], [15], [16], [17], [18] and terrestrial plasma technology (i.e. technology transfer) [19], [20], [21]. IPG have originally been developed to cope with chemically aggressive working gases for the IRS plasma wind tunnel PWK3. The electrodeless design enables additionally a pure plasma which engages the potential for aerothermochemical investigations in the field of heat shield material catalysis [8], [10], [22], nitridation and oxidation [23], [24] and, in addition, the behaviour of both plasma sources for plasma wind tunnels and electric propulsion and respective flow conditions [25]. Moreover, the high power inductively heated plasma sources developed at IRS were respectively characterized and modeled to provide increased understanding and an experimental database [4], [5], [26]. On basis of both system and mission analyses and the IPG-heritage, IPG6-S has been tested as IPT candidate in the context of ABEP [27], [28], [29], [30], [31].

\section{Literature Review of ABEP concepts}

Among the most advanced concepts for ABEP the most representative are those of JAXA, ESA, BUSEK, and SITAEL.

The Air-Breathing Ion Engine (ABIE), developed by Nishiyama, Fujita, and Hisamoto of the Japan Aerospace Exploration Agency (JAXA) [6]. The concept consists of an ECR (Electron Cyclotron Resonance) ion thruster. This type of thruster uses a microwave emitting antenna to generate an electric field and magnets to produce a strong magnetic field. A ring-shaped intake, surrounding the spacecraft core, is designed to provide a sufficient pressure to the thruster, estimated to be around 0.5 $\mathrm{Pa}$. Reflectors and a honeycomb structure of small ducts array at the intake entrance prevent the particles from exiting the intake in upstream direction. Designed for altitudes between 150 and $200 \mathrm{~km}$, it achieves a thrust density (thrust-to-power-ratio) of 10-13.7 mN/kW. The power requirement for full drag compensation at an altitude of $170 \mathrm{~km}$ is between $4-5.59 \mathrm{~kW}$.

A different approach was taken by ESA, presenting the RAM-EP Concept [9]. A medium-sized satellite was proposed with an estimated $\mathrm{C}_{\mathrm{d}}=2$, a cross sectional area of $1 \mathrm{~m}^{2}$ and a mass of $1000 \mathrm{~kg}$. This concept differs from ABIE in that the intake and thruster are physically separated. The RAM-EP is equipped with four RIT-10 to provide a combined thrust in the range of $2-20 \mathrm{mN}$ whilst the maximum power availability for propulsion is set to $1 \mathrm{~kW}$. The spacecraft is designed for a sun-synchronous orbit (SSO) at an altitude range of 180-250 km and has an estimated lifetime of 3-8 years. According to the study, at altitudes of more than $250 \mathrm{~km}$, conventional electric propulsion systems become more competitive, because 
of the lower drag and therefore lower propellant requirement, and because of the even more rarefied atmosphere that would deliver less mass flow to the RIT propulsion system. The intake is composed of a cylindrical array of diffusor baffles to increase pressure and prevent backflow.

The MABHET (Martian Atmosphere-Breathing HallEffect Thruster) concept by BUSEK [12] is an ABEP system designed for small spacecraft orbiting in around Mars. This concept consists of an intake, a Hall-effect thruster, and solar arrays as main power source, supported by batteries to cope with eclipses and power peaks. The thruster has been operated with a gas mixture similar to the Martian atmosphere (mostly $\mathrm{CO}_{2}$ ), achieving a thrust density of $19-33 \mathrm{mN} / \mathrm{kW}$, depending on the propellant mass flow. The intake is a cylinder measuring $3.7 \mathrm{~m}$ in length and $0.6 \mathrm{~m}$ in diameter, and allows a compression ratio of up to 100 and provides a collection efficiency of $\eta_{c}=35 \%$. Assuming a drag coefficient of $C_{d}=3,1.2 \mathrm{~kW}$ of power is estimated to be sufficient for full drag compensation at altitudes between $150-180 \mathrm{~km}$, which proves the general feasibility of this concept. According to [13], a maiden flight is planned for 2025 .

Shabshelowitz [18] examines the feasibility and efficiency of RF plasma technology regarding ABEP. The comparison of two thruster types, an RF plasma thruster (RPT) and a Helicon Hall Thruster (HHT), revealed that the utilisation of atmospheric gases, such as $\mathrm{N}_{2}$ and $\mathrm{O}_{2}$ leads to a lower efficiency of the HHT, in addition to the vulnerability regarding oxidation. The RPT produces less thrust operating with air and $\mathrm{N}_{2}$ than with Ar. The calculations and experiments are based on a concept spacecraft with a mass of $325 \mathrm{~kg}$, and an outer diameter of $0.7 \mathrm{~m}$. $\eta_{\mathrm{c}}$ is assumed to be $90 \%$. The required thrust at an orbit altitude of $200 \mathrm{~km}$ is estimated at 8.8 $\mathrm{mN}$ to achieve a mission lifetime of 3 years. To produce this amount of thrust using Ar, the RPT requires 1-1.5 $\mathrm{kW}$ of power depending on mass flow. With air, however, no thrust could be measured. The HHT requires $306 \mathrm{~W}$ of power, and was tested with $\mathrm{N}_{2}$.

SITAEL [19] proposes a concept based on an integrated RAM-EP concept including a Hall-effect thruster (HET). The system is designed for altitudes below $200 \mathrm{~km}$ and a lifetime of more than 4 years. The intake is built with a cylindrical array of ducts in a splitring configuration, and a conical convergent part at the back, which condenses the mass flow. The thruster is a HET with separate stages for ionisation and acceleration. The intake has a frontal area of $0.12 \mathrm{~m}^{2}$. The design altitude is $200 \mathrm{~km}$ and tests will be conducted with a corresponding mixture of $\mathrm{O}_{2}$ and $\mathrm{N}_{2}$. Ignition will be attempted with $\mathrm{Xe}$ or $\mathrm{Kr}$, and in the case of an insufficient mass flow will be assisted by a gas distributor downstream of the intake. The flow generator will be a $5 \mathrm{~kW}$ class Hall-effect thruster. SIATEL announced to have completed an end-to-end test of their system, however, as of September 2017, experimental results are not yet available.

\section{DISCOVERER ABEP concept}

This paper is realised within the context of the Europeanfunded project DISCOVERER which aims to revolutionise Earth-observation satellites for sustained operation at VLEO. IRS, in particular, has to develop an intake and an inductive plasma thruster (IPT) for an ABEP application.

The IPT is based on IPG6-S, a small-size IPG available at IRS. In an IPG a coil is wrapped around a quartz tube, the discharge channel, and fed by RF AC current. It operates in a way similar to a transformer where the primary winding is the coil and the secondary is the gas inside the discharge channel. The current flowing in the coil induces an oscillating magnetic field in the discharge channel which accelerates ions and electrons of the gas,

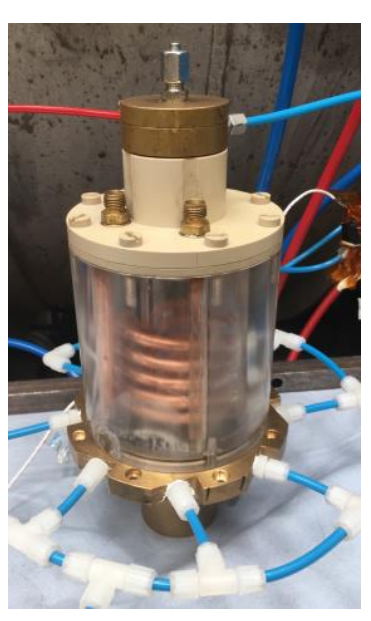

Fig. 2 IPG6-S plasma forms and a chain reaction establishes.

IPG6-S facility main parameters are: maximum input power $P_{\max }=20 \mathrm{~kW}$, maximum voltage of $1.7 \mathrm{kV}$, and variable frequency between $\mathrm{f}=3.5-4.5 \mathrm{MHz}$, depending on the impedance of the IPG. For the current configuration $\mathrm{f} \sim 4 \mathrm{MHz}$. IPG6-S, see Fig. 1, is water cooled, the discharge channel has an outer diameter of $40 \mathrm{~mm}$, a length of $180 \mathrm{~mm}$ and the coil has 5.5 turns providing an inductance of $0.489 \mathrm{H}$ [46]. A twin facility, IPG6-B, is installed at the University of Baylor, Waco, Texas, USA. A nozzle has been developed for IPG6-S in order to accelerate the plasma and produce thrust, paving the way for the development of the IPT. The nozzle is made of brass and water cooled, designed to be easily modified to allow various configurations: de Laval nozzle, convergent only, or no nozzle. The convergent section and the divergent section can be substituted to achieve various sizes and angles. The current design has a throat of $20 \mathrm{~mm}$ and the end of the divergent section again to 40 $\mathrm{mm}$. The nozzle is shown in Fig. 3. 
The current test facility for IPG6-S was previously used for RIT testing. The vacuum tank has a diameter of $2 \mathrm{~m}$ and a length of $\sim 5 \mathrm{~m}$, see Fig. 3. The main vacuum facility provides background pressure of $\sim 1$ $\mathrm{Pa}$, while a system of oil diffusion pumps can bring the background pressure down to $1 \mathrm{E}-5 \mathrm{~Pa}$. A recent

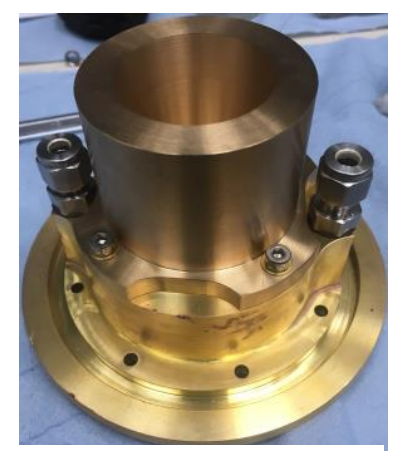

Fig. 3 de Laval nozzle refurbishment has been completed and preliminary tests will be performed by the first middle of September 2017. Test will include calorimeter measurements of the plasma plume energy leaving IPG6-S with various nozzle configuration.

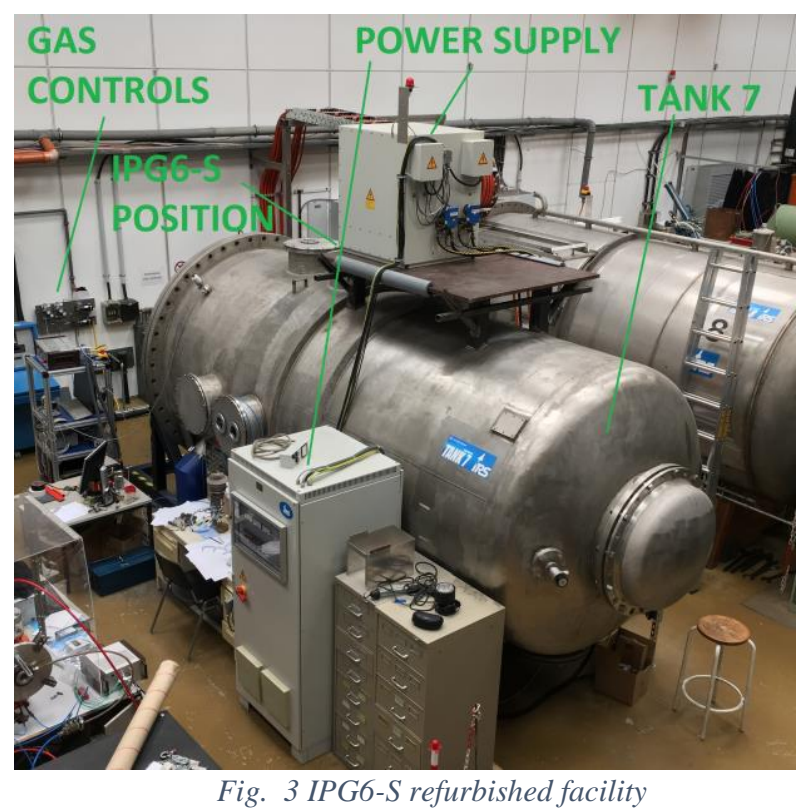

Concerning the intake, further analysis were done by IRS [23]-[25]. Intakes from ESA [1], BUSEK MABHET [2] and ABIE (JAXA) [3] were reviewed, and the last two analysed with DSMC for verification. A model based on simple algebraic relations was derived and verified by corresponding simulations. The common feature of the inlet section of all passive intake designs, is the idea of a "molecular trap" based on the transmittance characteristic for a flow of large speed ratio through an effectively long geometry, e.g. a tube or thin ring, with diffuse scattering afterwards. Simulations show that the transmittances depend only on $\mathrm{S}$ and the geometry, therefore the geometry can be optimised for the respective inflow conditions [25]. Another study [24] applied this balance model to a geometry based on the IPG6 discharge channel dimensions with $\mathrm{A}_{\text {out }}=1.075 \times 10^{-}$ ${ }^{3} \mathrm{~m}^{2}$. Calculations were done for an altitude of $140 \mathrm{~km}$ in
Earth atmosphere and $110 \mathrm{~km}$ in Mars atmosphere. Again, it was shown that an increasing area ratio Ain/Aout raises the collected mass flow, but reduces collection efficiency, which is equivalent to the thrust-to-drag-ratio of the spacecraft.

\section{Business Model}

According to [32] the starting point of innovative business models are its value propositions. [33] defined business model as a representation on how businesses operate. [34] defined the value proposition as the reason why a customer should buy a product or service from your business.

In this specific case, the innovative value proposition that ABEP systems may offer to the market can be expressed as "to enhance mission's lifetime and, accordingly, create more value for the investors". ABEP system is better than other EP drag-compensation systems, since it eliminates the weight of the propellant, increases the lifetime of the mission and, compared to other EP technologies, avoids the need for electrodes and their associated limitations. The next step in defining a business model is to identify the key resources and key activities. To take advantage of the ABEP value proposition, the key resources can be physicists and aerospace engineers that help in developing the electric thruster, and a plasma wind tunnel to test the system performance. Besides the key resources, the necessary key activities are research and testing to provide a better understanding of the use and performance of those innovative propulsion systems in LEO missions. Currently, ABEP systems are under study. They show very prominent advantages in front of other propulsion systems. However, the overall lifetime of the missions depends not only on the propulsion systems, but also on materials degradation. For that reason, further research in the elements that affect lifetime missions are required in order to be competitive in the market and get as much value as possible compared to the investments done.

\section{Conclusions}

The concept of ABEP has been depicted within this paper starting from a literature review and describing the advances done within the DISCOVERER project. Intake design. The nozzle has been designed, built, and ready to test for calorimeter plasma plume energy estimation. The refurbished facility is now ready for future IPG6-S and IPT development and testing.

\section{Acknowledgements}

F. Romano gratefully thanks the Landesgraduiertenf örderung of the University of Stuttgart for the financial support. 
$67^{\text {th }}$ International Astronautical Congress (IAC), Adelaide, Australia, 25-29 September 2017.

Copyright @ 2017 by the authors. Published by the International Astronautical Federation (IAF), with permission and released to the IAF to publish in all forms.

The DISCOVERER project has received funding from the European Union's Horizon 2020 research and innovation programme under grant agreement No 737183.

\section{References}

\section{List of references}

[1] ESA, GOCE System Critical Design Review (CDR), Tech. rep., ALENIA Spazio (May 2005). [2] J. V. Llop, P. Roberts, Z. Hao, L. R. Tomas, V. Beauplet, Very low earth's orbit mission concepts for Earth observation. benefits and challenges, in: 12th Reinventing Space Conference, London, United Kingdom, Vol. $\quad 520, \quad 2014$. [3] G. Herdrich, M. Fertig, S. Löhle, Experimental simulation of high enthalpy planetary entries, The Open Journal of Plasma Physics 2, ISSN: 1876-5343 (2009) $150\{164$ (15). doi:10.2174/1876534300902010150. [4] G. Herdrich, D. Petkow, Water-cooled and thinwalled ICP sources: Characterization and MHDoptimization, Journal of Plasma Physics 74 (3) (2008) $391\{429$. doi:10.1017/S0022377807006927.

[5] B. Massuti-Ballester, T. Marynowski, G. Herdrich, New inductively heated source IPG7, Frontiers of Applied Plasma Technology 7 (1) (2014) 1-5. [6] R. Wernitz, C. Eichhorn, T. Marynowski, G. Herdrich, Plasma wind tunnel investigation of european ablators in nitrogen/methane using emission spectroscopy, Hindawi International Journal of Spectroscopy 2013 Article ID $764321 \quad$ (2013) 9. doi:10.1155/2013/764321. [7] Y. Kubota, K. Fukuda, H. Hatta, R. Wernitz, G. Herdrich, S. Fasoulas, Comparison of thermal deformations of carbon fiber-reinforced phenolic matrix ablators by arc-plasma wind tunnel heating and quasistatic heating, Advanced Composite Materials 4;24(2) (2015) 179-195. doi:10.1080/09243046.2014.882539.

[8] B. Massuti-Ballester, S. Pidan, G. Herdrich, M. Fertig, Recent catalysis measurements at IRS, Advances in Space Research 56 (4) (2015) 742-765. doi:http://dx.doi.org/10.1016/j.asr.2015.04.028.

[9] A. S. Pagan, B. Massuti-Ballester, G. Herdrich, Total and spectral emissivities of demising aerospace materials, Frontier of Applied Plasma Technology 9 (1) (2016) 713.

[10] N. Joiner, B. Esser, M. Fertig, A. Gülhan, G. Herdrich, B. Massuti-Ballester, Development of an innovative validation strategy of gas-surface interaction modelling for re-entry applications, CEAS Space Journal 8 (4) (2016) 237-255. doi:10.1007/s12567-016-0124-6. [11] M. Schüßler, M. Auweter-Kurtz, G. Herdrich, S. Lein, Surface characterization of metallic and ceramic tps-materials for reusable space vehicles, Acta Astronautica $65 \quad(5\{6) \quad$ (2009) 676-686. doi:http://dx.doi.org/10.1016/j.actaastro.2009.01.048.
[12] G. Herdrich, U. Bauder, D. Bock, C. Eichhorn, M. Fertig, D. Haag, M. Lau, T. Schönherr, T. Stindl, H.-P. Röser, M. Auweter-Kurtz, Activities in electric propulsion development at IRS, Invited Talk-Paper 2008-b-02, Selected papers from the 26th International Symposium on Space Technology and Science, Transactions of Japan Society for Aeronautical and Space Sciences 7 (ists26) (2009) Tb5-Tb14. [13] C. Syring, G. Herdrich, Jet extraction modes of inertial electrostatic confinement devices for electric propulsion applications, Vacuum 136 (2017) 177-183. [14] B. Wollenhaupt, Q. H. Le, G. Herdrich, An overview about international thermal arcjet thruster development, accepted by Emerald Aircraft Engineering and Aerospace Technology doi:10.1108/AEAT-08-20160124.R2.

[15] M. Lau, S. Manna, G. Herdrich, T. Schönherr, K. Komurasaki, Investigation of the plasma current density of a pulsed plasma thruster, Journal of Propulsion and Power 30 (6) (2014) 1459-1470. doi:10.2514/1.B35131. [16] A. Boxberger, G. Herdrich, L. Malacci, F. D. de Mendoza Alegre, Overview of experimental research on applied-field magnetoplasmadynamic thrusters at IRS, 5th Russian-German Conference on Electric Propulsion, Dresden, Germany. [17] G. Herdrich, U. Bauder, A. Boxberger, R. Gabrielli, M. Lau, D. Petkow, M. Pfeiffer, C. Syring, S. Fasoulas, Advanced plasma (propulsion) concepts at IRS, Vacuum $\begin{array}{llll}\text { Journal } & 88 & \text { (2012) } & \text { 36-41. }\end{array}$ doi:10.1016/j.vacuum.2012.02.032.

[18] A. R. Chadwick, G. Herdrich, M. K. Kim, B. Dally, Transient electromagnetic behaviour in inductive oxygen and argon-oxygen plasmas, Plasma Sources Science and $\begin{array}{lllll}\text { Technology } & 25 & \text { (6) } & \text { (2016) } & 065025\end{array}$ [19] G. Herdrich, M. Fertig, D. Petkow, S. Kraus, S. L"ohle, M. Auweter-Kurtz, Operational behavior and application regime assessment of the magnetic acceleration plasma facility IMAX, Vacuum Journal 85 (2010) 563-568. doi:10.1016/j.vacuum.2010.08.012. [20] D. Hoffmann, M. Mueller, G. Herdrich, D. Petkow, S. Lein, Experimental investigation of a capacitive blind hollow cathode discharge with central gas injection, Plasma Sources Science and Technology 23 (6) (2014) 1459-1470. doi:10.1088/0963-0252/23/6/065023. [21] G. Herdrich, M. Auweter, Inductively heated plasma sources for technical applications, Vacuum Journal, Institut für Raumfahrtsysteme (IRS) and Steinbeis Transfer Centre Plasma and Space Technology (STC PRT) $\quad 80 \quad$ (2006) 1138-1143. [22] G. Herdrich, M. Fertig, D. Petkow, A. Steinbeck, S. Fasoulas, Experimental and numerical techniques to assess catalysis, Progress in Aerospace Sciences 48-49 (2012) 27-41. doi:10.1016/j.paerosci.2011.06.007. [23] G. Herdrich, M. Auweter-Kurtz, M. Fertig, S. Löhle, S. Pidan, T. Laux, Oxidation behaviour of SiC-based thermal protection system materials using newly 
developed probe techniques, AIAA meeting papers on disc, (2004-2173), American Institute of Aeronautics and Astronautics, [Reston, Va.] 42 (5) (2005) 817-824. [24] M. Fertig, G. Herdrich, The advanced URANUS Navier-Stokes code for the simulation of nonequilibrium re-entry flows, Transactions of Japan Society for Aeronautical and Space Sciences, Space Technology

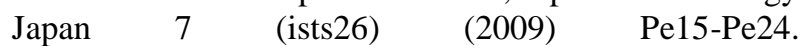
[25] D. Petkow, G. Herdrich, M. Pfeiffer, A. Mirza, S. Fasoulas, M. Matsui, K. Komurasaki, On the probabilistic particle simulation of an arcjet flow expansion, Vacuum Journal 88 (2013) 58-62. doi:10.1016/j.vacuum.2012.04.047.

[26] G. Herdrich, T. Marynowski, M. Dropmann, S. Fasoulas, Mars and Venus entry simulation capabilities of IRS plasma wind tunnel PWK3, Applied Physics Research 4 (1) (2012) 146-155. doi:10.5539/apr.v4n1p146.

[27] T. Schönherr, K. Komurasaki, F. Romano, B. Massuti-Ballester, G. Herdrich, Analysis of atmospherebreathing electric propulsion, Plasma Science, IEEE Transactions on 43 (1) (2015) 287\{294. doi:10.1109/TPS.2014.2364053.

[28] F. Romano, B. Massuti-Ballester, T. Schönherr, G. Herdrich, System analysis and test bed for an airbreathing electric propulsion system, in: 5th Russian-
German Conference on Electric Propulsion (RGCEP), Dresden, Germany, 2014 [29] F. Romano, T. Binder, G. Herdrich, S. Fasoulas, T. Schönherr, Air-intake design investigation for an airbreathing electric propulsion system, 34th International Electric Propulsion Conference, Kobe, Japan IEPC2015/ISTS-2015-b

(269). [30] F. Romano, T. Binder, G. Herdrich, S. Fasoulas, T. Schönherr, Intake design for an atmosphere-breathing electric propulsion system, Space Propulsion 2016, Roma, Italy, 2016 SP2016 (3124981). [31] T. Binder, P. Boldini, F. Romano, G. Herdrich, S. Fasoulas, Transmission probabilities of rarefied flows in the application of atmosphere-breathing electric propulsion, in: AIP Conference Proceedings, Vol. 1786, AIP Publishing, 2016, p. 190011. doi:10.1063/1.4967689. [32] P. Kesting, F. Günzel-Jensen, "SMEs and new ventures need business model sophistication", Business Horizons, Volume 58, Issue 3, May-June 2015, Pages 285-293

[33] Magretta, J. "Why Business Models Matter," Harvard Business Review, May 2002

[34] Wilson, H.N.; Macdonald, E.K. and Baxendale, S. "What really makes customers buy a product", Harvard Business Review, November 2015 